\title{
BMJ Open Low-value clinical practices in adult traumatic brain injury: an umbrella review protocol
}

Pier-Alexandre Tardif (D) , ${ }^{1}$ Lynne Moore, ${ }^{1,2}$ François Lauzier, ${ }^{1,3}$ Imen Farhat, ${ }^{1,2}$ Patrick Archambault, ${ }^{1,4}$ Francois Lamontagne, ${ }^{5}$ Michael Chassé, ${ }^{6}$ Henry Thomas Stelfox, ${ }^{7}$ Belinda J Gabbe, ${ }^{8,9}$ Fiona Lecky, ${ }^{10}$ John Kortbeek, ${ }^{11}$ Paule Lessard-Bonaventure, ${ }^{1,12}$ Catherine Truchon, ${ }^{13}$ Alexis F Turgeon (D) ${ }^{1,3}$

To cite: Tardif P-A, Moore L, Lauzier $\mathrm{F}$, et al. Lowvalue clinical practices in adult traumatic brain injury: an umbrella review protocol. BMJ Open 2019;9:e031747. doi:10.1136/ bmjopen-2019-031747

- Prepublication history for this paper is available online. To view these files, please visit the journal online (http://dx.doi org/10.1136/bmjopen-2019031747).

Received 16 May 2019 Revised 06 August 2019 Accepted 04 September 2019

D Check for updates

(c) Author(s) (or their employer(s)) 2019. Re-use permitted under CC BY-NC. No commercial re-use. See rights and permissions. Published by BMJ.

For numbered affiliations see end of article.

\section{Correspondence to} Pier-Alexandre Tardif; Pier-alexandre.tardif@ crchudequebec.ulaval.ca

\section{ABSTRACT}

Introduction Traumatic brain injury (TBI) leads to 50000 deaths, 85000 disabilities and costs $\$ 60$ billion each year in the USA. Despite numerous interventions and treatment options, the outcomes of TBI have improved little over the last three decades. In a previous scoping review and expert consultation survey, we identified 13 potentially low-value clinical practices in acute TBI. The objective of this umbrella review is to synthesise the evidence on potentially low-value clinical practices in the care of acute TBI.

Methods and analysis Using umbrella review methodology, we will search Cochrane Central Register of Controlled Trials, Embase, Epistemonikos, International Prospective Register of Systematic Reviews (PROSPER0) and PubMed to identify systematic reviews evaluating the effect of potential intrahospital low-value practices using tailored population, intervention, comparator, outcome and study design questions based on the results of a previous scoping review. We will present data on the methodological quality of these reviews (Assessing the Methodological Quality of Systematic Reviews-2), reported effect sizes and strength of evidence (Grading of Recommendations, Assessment, Development and Evaluation).

Ethics and dissemination Ethics approval is not required as original data will not be collected. Knowledge users from five healthcare quality organisations and clinical associations are involved in the design and conduct of the study. Results will be disseminated in a peer-reviewed journal, at international scientific meetings and to clinical, healthcare quality and patient-partner associations. This work will support the development of metrics to measure the use of low-value practices, inform policy makers on potential targets for deimplementation and in the long term reduce the use of low-value clinical practices in acute TBI care.

PROSPERO registration number CRD42019132428.

\section{INTRODUCTION}

Traumatic brain injury (TBI) is the main cause of mortality from injury in people under 45 years of age, ${ }^{1}$ and it leads to approximately US $\$ 60$ and $€ 33$ billion in total medical costs in the $\mathrm{USA}^{2}$ and Europe ${ }^{3}$ each year, respectively. Moreover, outcomes

\section{Strengths and limitations of this study}

- State-of-the-art synthesis of evidence on low-value clinical practices in the care of acute traumatic brain injury (TBI).

- Represents a crucial step towards the deimplementation of low-value practices in acute TBI care.

- Adopts an integrated knowledge translation model to ensure the results are relevant to decision makers.

- For feasibility reasons, our synthesis is restricted to systematic reviews published in English since 1990.

- The scope of review and the inclusion of systematic reviews precludes meta-analysis.

following TBI have not improved significantly in the last four decades. ${ }^{45}$ Intervention and treatment options for TBI are multiple, but many lack robust evidence of their effectiveness. ${ }^{6} 7$

Low-value clinical practices, defined as a test or procedure that is not supported by evidence and/or could expose patients to unnecessary harm ${ }^{8-15}$ consume up to $30 \%$ of healthcare budgets. ${ }^{9}{ }^{16}$ In the past decade, the medical community has turned towards the deimplementation of low-value practices as a promising means to reduce the strain on healthcare budgets, free-up resources and reduce harm to patients. ${ }^{17}$ Physicians report using low-value practices because of a lack of alternative treatment options, fear of legal consequences but also because of lack of guidelines on low-value care. ${ }^{15} 18$ The Brain Trauma Foundation, among others, publish guidelines on TBI care. ${ }^{19}$ However, emphasis is on practices that should be adhered to rather than practices that should be avoided. Choosing Wisely publish recommendations specifically targeting low-value practices but few pertain to TBI care and many are based uniquely on expert consensus. ${ }^{11}$ A previous 
scoping review and expert consultation survey identified 13 potentially low-value clinical practices in acute TBI care. ${ }^{20}$ These practices represent potential targets for guidelines, overuse metrics and deimplementation interventions. However, before recommendations can be made, we need to synthesise the evidence base for these practices.

Interventions and treatment options for acute TBI have been the subject of multiple systematic reviews. ${ }^{2122}$ Given this large body of available evidence, evidence maps have previously been used to summarise evidence from systematic reviews on acute TBI interventions. ${ }^{723}$ However, these evidence maps were not designed to target low-value practices and focused on moderate to severe TBI when the mild TBI population represent great potential for reducing low-value care. In addition, previous reviews have not provided a synthesis of effect sizes or strength of evidence. The objective of the present study is to synthesise the evidence on potentially low-value intrahospital clinical practices in acute adult TBI.

\section{METHODS AND ANALYSIS}

Given the multitude of systematic reviews available for the clinical practices identified as potentially low-value (over 60 were identified in our scoping review), we opted to conduct an umbrella review (a systematic review of systematic reviews) ${ }^{20}$ While the former aimed to fill a knowledge gap on medical overuse for acute injury care by identifying all potential low-value clinical practices, the latter will synthesise the evidence on the low-value practices pertaining to TBI. The review will be conducted according to published guidelines. ${ }^{24-26}$ In the absence of reporting guidelines for umbrella reviews, we will use the applicable Preferred Reporting Items for Systematic Reviews and Meta-Analysis (PRISMA) Protocols. ${ }^{27}$

\section{Eligibility criteria}

The project steering committee comprising clinicians (two emergency physicians, seven critical care physicians, one neurosurgeon), methodologists (four) and health system managers (three) used the population, intervention, comparator, outcome and study design (PICOS) framework to develop specific research questions for each potentially low-value clinical practice (table 1). ${ }^{20} 28$ We will consider systematic reviews of original studies evaluating the effectiveness of predetermined clinical practices in acute TBI in adults $(\geq 16$ years old) without restriction on location of publication but limited to studies published in English since $1990 . .^{2526}$

We will use the Cochrane definition to identify systematic reviews. We will consider a review to be systematic if it clearly stated a set of objectives and reported explicit eligibility criteria, an extensive search strategy (a refined search strategy ran on Medline or Cochrane Library
Table 1 PICOS for each clinical practice

\section{\# Clinical practice}

Mild traumatic brain injury

1 Population: adults with acute mild traumatic brain injury

Intervention: validated clinical decision rule (eg,

CCHR, CHIP, NEXUS II, NOC)

Comparator: none

Primary outcome: false negative rate (intracranial injury, neurosurgical intervention)

Secondary outcomes: sensitivity, specificity

Study design: systematic review

2 Population: adults with acute mild complicated traumatic brain injury

Intervention: routine repeat head CT in absence of neurological deterioration

Comparator: none or no repeat head CT in absence of neurological deterioration

Primary outcome: progression of intracranial injury Secondary outcomes: neurosurgical intervention, mortality, change in management, hospital length of stay

Study design: systematic review

3 Population: adults with acute mild traumatic brain injury and on anticoagulant and/or antiplatelet therapy Intervention: routine repeat head CT in absence of neurological deterioration

Comparator: none or no repeat head CT in absence of neurological deterioration

Primary outcome: progression of intracranial injury Secondary outcomes: neurosurgical intervention, mortality, change in management, hospital length of stay

Study design: systematic review

4 Population: adults with acute mild traumatic brain injury who are negative on head CT Intervention: neurosurgical consultation Comparator: none or no neurosurgical consultation Primary outcome: hospital admission Secondary outcomes: neurosurgical intervention, mortality, ICU admission, repeat head CT, hospital length of stay Study design: systematic review

5 Population: adults with acute mild complicated traumatic brain injury who are not on irreversible anticoagulation

Intervention: intensive care unit admission

Comparator: admission to regular ward or stepdown unit

Primary outcome: neurological/medical decline, neurosurgical intervention

Secondary outcomes: medical interventions, mortality, adverse events, hospital length of stay, discharge destination Study design: systematic review

Moderate and severe traumatic brain injury 


\begin{tabular}{ll} 
Table 1 & Continued \\
\hline$\# \quad$ & Clinical practice \\
\hline 6 & Population: adults with acute traumatic brain injury \\
& on antiplatelet therapy \\
& Intervention: platelet transfusion \\
& Comparator: no platelet transfusion \\
& Primary outcome: GOS or GOS-E \\
& Secondary outcomes: mortality, adverse events, \\
& hospital and ICU length of stay \\
& Study design: systematic review \\
& Population: adults with basal skull fractures without \\
& evidence of cerebrospinal fluid leakage \\
Intervention: antibiotic prophylaxis & \\
Comparator: no antibiotic prophylaxis \\
Primary outcome: meningitis (confirmed by lumbar \\
puncture) \\
Secondary outcomes: GOS or GOS-E, mortality, \\
surgical correction in patients with CSF leakage, \\
non-CNS infection, hospital and ICU length of stay \\
Study design: systematic review \\
Population: adults with acute traumatic brain injury \\
and no refractory intracranial hypertension \\
Intervention: therapeutic hypothermia \\
Comparator: no therapeutic hypothermia \\
Primary outcome: GOS or GOS-E \\
Secondary outcomes: intracranial pressure, \\
mortality, adverse events, hospital and ICU length of \\
stay \\
Study design: systematic review
\end{tabular}

9 Population: adults with acute traumatic brain injury Intervention: antibiotic prophylaxis for external ventricular drain placement

Comparator: no antibiotic prophylaxis

Primary outcome: ventriculostomy-related infection Secondary outcomes: GOS, mortality, hospital and ICU length of stay

Study design: systematic review

10 Population: adults with acute traumatic brain injury and no refractory intracranial hypertension Intervention: neuromuscular blocking agents Comparator: no neuromuscular blocking agents Primary outcome: GOS or GOS-E

Secondary outcomes: intracranial pressure, mortality, adverse events, hospital and ICU length of stay

Study design: systematic review

Population: adults with acute traumatic brain injury
Intervention: plasma transfusion
Comparator: no plasma transfusion
Primary outcome: GOS or GOS-E
Secondary outcomes: mortality, adverse events,
hospital and ICU length of stay
Study design: systematic review

Severe traumatic brain injury

Continued

\begin{tabular}{|c|c|}
\hline$\#$ & Clinical practice \\
\hline 12 & $\begin{array}{l}\text { Population: adults with acute severe traumatic brain } \\
\text { injury } \\
\text { Intervention: albumin } \\
\text { Comparator: any other colloid-containing fluids } \\
\text { (dextrans, modified gelatins, hydroxyethyl starches) } \\
\text { or isotonic crystalloid fluids (saline } 0.9 \% \text { and } \\
\text { balanced salt solutions such as compound sodium } \\
\text { lactate, Plasma-Lyte) } \\
\text { Primary outcome: GOS or GOS-E } \\
\text { Secondary outcomes: mortality, adverse events, } \\
\text { hospital and ICU length of stay } \\
\text { Study design: systematic review }\end{array}$ \\
\hline 13 & $\begin{array}{l}\text { Population: adults with acute severe traumatic brain } \\
\text { injury } \\
\text { Intervention: antiseizure prophylaxis (levetiracetam } \\
\text { or phenytoin)>1 week } \\
\text { Comparator: antiseizure prophylaxis < } 1 \text { week or no } \\
\text { antiseizure prophylaxis } \\
\text { Primary outcome: late post-traumatic seizure } \\
\text { Secondary outcomes: GOS or GOS-E, mortality, } \\
\text { adverse events, hospital and ICU length of stay } \\
\text { Study design: systematic review }\end{array}$ \\
\hline
\end{tabular}

CCHR, Canadian Computed Tomography Head Rule; CHIP, CT in head injury patients; CNS, central nervous system; CSF, cerebrospinal fluid; GOS, Glasgow Outcome Scale; GOS-E, Glasgow Outcome Scale-Extended; ICU, intensive care unit; NEXUS, National Emergency X-Radiography Utilisation Study; NOC, New Orleans Criteria; PICOS, population, intervention, comparator, outcome and study design.

and at least one other database $)^{29}{ }^{30}$ and reproducible methods to identify, select and critically appraise the findings of the included studies. ${ }^{24}$

\section{Outcomes}

Primary and secondary outcomes were identified for each of the evaluated clinical practices by the project steering committee and are described in a PICO format in table 1.

\section{Search strategy}

In consultation with an information specialist, we will develop comprehensive literature search strategies separately for each clinical practice to be studied (see table 2 for a preliminary search strategy in PubMed). We will search systematic reviews using the Cochrane Library, Excerpta Medica Database (Embase), Epistemonikos, ${ }^{31}$ PubMed and the International Prospective Register of Systematic Reviews ${ }^{32}$ from 1990 to up to 6 months prior to submission for publication. Using a snowball approach, we will screen the references of included studies in addition to previous reviews on this subject. ${ }^{71-2333}$

\section{Selection process}

We will manage all citations with EndNote software V.X8.2 (Clarivate Analytics, 2014). We will identify and remove duplicates using electronic and manual screening. ${ }^{34}$ To ensure reliability when selecting studies for a given 


\begin{tabular}{|c|c|c|}
\hline Concepts & PubMed search strategy & Research \\
\hline Injury & $\begin{array}{l}\text { "Craniocerebral Trauma"[Majr] OR [diffus* AND axonal injur*[Title/Abstract]] OR } \\
\text { "head trauma"[Title/Abstract]OR "head injury"[Title/Abstract]OR "head injuries"[Title/ } \\
\text { Abstract]OR "brain trauma"[Title/Abstract]OR "brain injury"[Title/Abstract]OR "brain } \\
\text { injuries"[Title/Abstract]OR "cerebral trauma"[Title/Abstract]OR "cerebral injury"[Title/ } \\
\text { Abstract]OR "cerebral injuries"[Title/Abstract]OR "craniocerebral trauma"[Title/Abstract] } \\
\text { OR "craniocerebral injury"[Title/Abstract]OR "craniocerebral injuries"[Title/Abstract] } \\
\text { OR "TBI"[Title/Abstract]OR "traumatic brain injury"[Title/Abstract]OR "traumatic brain } \\
\text { injuries"[Title/Abstract]OR "brainstem trauma"[Title/Abstract]OR "brainstem injury"[Title/ } \\
\text { Abstract]OR "brainstem injuries"[Title/Abstract]OR "Head Injuries, Closed"[MeSH:NoExp] } \\
\text { OR "Brain Injuries"[MeSH:NoExp]OR "Craniocerebral Trauma"[MeSH:NoExp]OR "Brain } \\
\text { Hemorrhage, Traumatic"[MeSH] OR "Diffuse Axonal Injury"[MeSH:NoExp]OR "Coma, Post- } \\
\text { Head Injury"[MeSH:NoExp]OR "Head Injuries, Penetrating"[MeSH:NoExp]OR "Intracranial } \\
\text { Hemorrhage, Traumatic"[MeSH] OR "Skull Fractures"[MeSH] }\end{array}$ & $\# 1$ \\
\hline Clinical practice & $\begin{array}{l}\text { "Hypothermia"[Mesh] OR "Cryotherapy"[Mesh] OR "Body Temperature"[Mesh] OR "artificial } \\
\text { hibernation"[Title/Abstract]OR "body cooling"[Title/Abstract]OR cold*[Title/Abstract]OR } \\
\text { cool*[Title/Abstract]OR "cooling therapy"[Title/Abstract]OR "cooling therapies"[Title/Abstract] } \\
\text { OR cryogen*[Title/Abstract]OR cryother*[Title/Abstract]OR cryotreat*[Title/Abstract]OR } \\
\text { hypotherm*[Title/Abstract]OR normotherm[Title/Abstract]OR refrigeration*[Title/Abstract]OR } \\
\text { temperature*[Title/Abstract] }\end{array}$ & \#2 \\
\hline $\begin{array}{l}\text { Filter for } \\
\text { systematic } \\
\text { reviews }\end{array}$ & $\begin{array}{l}\text { (((systematic review[ti] OR systematic literature review[ti] OR systematic scoping review[ti] OR } \\
\text { systematic narrative review[ti] OR systematic qualitative review[ti] OR systematic evidence } \\
\text { review[ti] OR systematic quantitative review[ti] OR systematic meta-review[ti] OR systematic } \\
\text { critical review[ti] OR systematic mixed studies review[ti] OR systematic mapping review[ti] OR } \\
\text { systematic cochrane review[ti] OR systematic search and review[ti] OR systematic integrative } \\
\text { review[ti]] NOT comment[pt] NOT [protocol[ti] OR protocols[ti]]) NOT MEDLINE [subset]) OR } \\
\text { (Cochrane Database Syst Rev[ta] AND review[pt)] OR systematic review[pt] }\end{array}$ & \#3 \\
\hline Total & \#1 AND \#2 AND \#3 & \#4 \\
\hline
\end{tabular}

practice, two sets of 100 citations will independently be evaluated and then discussed by the reviewers. Pairs of reviewers (PAT, LM, IF, KMB) will then independently screen all identified records using titles, abstracts and full texts, consecutively. Any disagreement will be resolved through discussion between reviewers and, if necessary, consultation with a senior author (AFT). Potentially eligible studies excluded using full texts will be described in a PRISMA flow chart.

\section{Data items and abstraction process}

Using a standardised data abstraction form piloted on a representative sample of five studies, pairs of experienced reviewers (PAT, LM, IF, KMB) will independently extract the following data: first author, title, year of publication, databases used and date of the last search; population(s), intervention(s), comparator(s), outcome(s) and study designs included; measures of association and their respective measure of heterogeneity; tools used to assess the quality (risk of bias) of original studies and overall rating from the authors. Any disagreement will be resolved through discussion between reviewers and, if necessary, consultation with a senior author (AFT). When information is available in figures only, we will abstract graphical data using computer-assisted software. ${ }^{35} 36$ Furthermore, we will contact study authors (up to three email attempts) when information is unclear or unavailable.

\section{Methodological quality assessment}

Two reviewers (PAT, LM) will independently critically appraise the quality of systematic reviews using the Assessing the Methodological Quality of Systematic Reviews (AMSTAR-2) tool. ${ }^{37}$ Methodological quality will be categorised as low (0-3), medium (4-7) and high (8-11).

\section{Synthesis}

Results will be presented according to current recommendations for umbrella reviews. ${ }^{38}$ For each low-value practice, we will present the number of studies, study designs and patients included, the quality of the reviews (AMSTAR-2), effect sizes for primary and secondary outcomes (forest plots) and strength of recommendations (Grading of Recommendations, Assessment, Development and Evaluation).

\section{Potential limitations}

To ensure the feasibility of the review, we will restrict our search to low-value practices identified in the scoping review and expert consultation study, which may lead us to miss some low-value practices. However, given the robust search strategy used in our scoping review and the fact that experts were asked to add any other practices they considered low value, it is unlikely that important low-value practices have been missed. By targeting systematic reviews rather than original studies, we may miss some 
evidence. However, given the availability of high-quality, up-to-date reviews in TBI care suggested by our scoping review, we think it unlikely that we will miss a large body of evidence. For certain clinical practices, we may not identify any high-quality, up-to-date reviews. These practices will be the subject of systematic reviews in subsequent phases of the research programme. Finally, for feasibility reasons, we limited this umbrella review to reviews published in English since 1990 as per recommendations for umbrella reviews. ${ }^{25}{ }^{26}$ These limitations should have negligible impact on results since few systematic reviews were published prior to 1990 and most published reviews are likely to be written in English. ${ }^{25} 26$

\section{Potential impact}

This review is part of the Canadian Program on Monitoring Low-Value Clinical Practices in Injury Care (Canadian Institutes of Health Research \#113664), aiming to evaluate the effectiveness of an audit-feedback module targeting low-value clinical practices in acute injury care. The results of this review will be used to inform the development of quality indicators to be integrated in the audit-feedback module.

We will use state-of-the-art methods to optimise the sensitivity of our search strategy and the robustness of results. Results will be synthesised graphically. Ultimately, this research will inform the development of metrics, guidelines and deimplementation interventions, all targeting low-value injury care. The reduction of low-value clinical practices in acute TBI care has the potential to reduce pressure on strained healthcare budgets, free up resources, reduce adverse events and improve patient outcomes.

\section{Ethics and dissemination}

Ethics approval is not required as original data will not be collected. This study will be disseminated in a peer-reviewed journal, international scientific meetings, to knowledge users through clinical and healthcare quality associations (Choosing Wisely Canada, Trauma Association of Canada, American College of SurgeonsCommittee on Trauma, International Federation of Emergency Medicine, Institut national d'excellence en santé et en services sociaux, Brain Trauma Foundation) and to patient partners associations (Brain Injury Canada).

\section{Patient and public involvement}

No patient or public representatives will be involved in this study.

\footnotetext{
Author affiliations

${ }^{1}$ Population Health and Optimal Health Practices Unit, Trauma-EmergencyCritical Care Medicine, Centre de Recherche du CHU de Québec-Université Laval (Hôpital de l'Enfant-Jésus), Université Laval, Montreal, Quebec, Canada ${ }^{2}$ Department of Social and Preventative Medicine, Université Laval, Montreal, Quebec, Canada

${ }^{3}$ Division of Critical Care Medicine, Department of Anesthesiology and Critical Care Medicine, Université Laval, Quebec City, Quebec, Canada

${ }^{4}$ Département de médecine familiale et médecine d'urgence, Université Laval,

Québec, QC, Canada

${ }^{5}$ Department of Medicine, Université de Sherbrooke, Sherbrooke, Quebec, Canada
}

${ }^{6}$ Department of Medicine, Université de Montréal, Montreal, Quebec, Canada ${ }^{7}$ Departments of Critical Care Medicine, Medicine and Community Health Sciences, 0'Brien Institute for Public Health, University of Calgary, Calgary, Alberta, Canada ${ }^{8}$ Monash University School of Public Health and Preventive Medicine, Melbourne, Victoria, Australia

${ }^{9}$ Farr Institute, Swansea University Medical School, Swansea University, Swansea, UK

${ }^{10}$ School of Health and Related Research, The University of Sheffield, Sheffield, UK

${ }^{11}$ Department of Surgery, University of Calgary, Calgary, Alberta, Canada

${ }^{12}$ Department of Surgery, Division of Neurosurgery, Université Laval, Quebec City, Quebec, Canada

${ }^{13}$ Institut national d'excellence en santé et en services sociaux du Québec, Quebec City, Quebec, Canada

Contributors P-AT contributed to the elaboration of keywords, developed and tested the search strategy, drafted the manuscript and approved the final version of the manuscript. LM led the development of the protocol and drafted the manuscript with the first author. She acts as guarantor for the review. FLau contributed to developing keywords, validated the search strategy and the data extraction form, revised the manuscript and approved the final version. IF contributed to the elaboration of keywords, the search strategy and the data extraction form, critically revised and approved the final version of the manuscript. PA contributed to working definitions, developed keywords, revised the manuscript and approved the final version. FLam contributed to working definitions, developed keywords, revised the manuscript and approved the final version. MC validated the search strategy and the data extraction form, revised the manuscript and approved the final version. HTS contributed to the development of research objectives, inclusion criteria, the search strategy and the extraction form, developed keywords, revised the manuscript and approved the final version. BJG elaborated inclusion and exclusion criteria and keywords, revised the manuscript and approved the final version. FLec elaborated inclusion and exclusion criteria and keywords, revised the manuscript and approved the final version. JK contributed to the development of research objectives, study definitions, inclusion criteria and the extraction form, developed keywords, revised the manuscript and approved the final version. PL-B contributed to the development of research objectives and inclusion criteria, elaborated keywords, validated the data extraction form, critically revised the manuscript and approved the final version. CT elaborated inclusion and exclusion criteria and keywords, contribution to the development of the conceptual framework and concept definitions, revised the manuscript and approved the final version. AFT elaborated inclusion criteria and clinically significant outcomes, validated the search strategy, elaborated keywords, revised the manuscript and approved the final version.

Funding This research was supported by the Canadian Institutes of Health Research (Foundation grant, \#353374 and Embedded Clinician Researcher (PA)). LM, FLau, FLam and MC are recipients of a research salary award from the Fonds de Recherche du Québec-Santé (FRQS). AFT is the Canada Research Chair in Critical Care Neurology and Trauma. The funders had no role in developing this protocol.

Competing interests None declared.

Patient consent for publication Not required.

Provenance and peer review Not commissioned; externally peer reviewed.

Open access This is an open access article distributed in accordance with the Creative Commons Attribution Non Commercial (CC BY-NC 4.0) license, which permits others to distribute, remix, adapt, build upon this work non-commercially, and license their derivative works on different terms, provided the original work is properly cited, appropriate credit is given, any changes made indicated, and the use is non-commercial. See: http://creativecommons.org/licenses/by-nc/4.0/.

ORCID iDs

Pier-Alexandre Tardif http://orcid.org/0000-0003-3003-2399

Alexis F Turgeon http://orcid.org/0000-0001-5675-8791

\section{REFERENCES}

1. The American Association for the Surgery of Trauma (AAST). Traumatic brain injury in the United States: a report to Congress, CDC, 1999. Available: http://www.aast.org/trauma-facts [Accessed 19 Nov 2018]. 
2. Coronado VG, Haileyesus T, Cheng TA, et al. Trends in Sports- and Recreation-Related traumatic brain injuries treated in US emergency departments: the National electronic injury surveillance System-All injury program (NEISS-AIP) 2001-2012. J Head Trauma Rehabil 2015;30:185-97.

3. Olesen J, Gustavsson A, Svensson M, et al. The economic cost of brain disorders in Europe. Eur J Neurol 2012;19:155-62.

4. Rosenfeld JV, Maas Al, Bragge P, et al. Early management of severe traumatic brain injury. The Lancet 2012;380:1088-98.

5. Maas AIR, Menon DK, Adelson PD, et al. Traumatic brain injury: integrated approaches to improve prevention, clinical care, and research. Lancet Neurol 2017;16:987-1048.

6. Maas AIR, Roozenbeek B, Manley GT. Clinical trials in traumatic brain injury: past experience and current developments. Neurotherapeutics 2010;7:115-26.

7. Bragge P, Synnot A, Maas Al, et al. A State-of-the-Science overview of randomized controlled trials evaluating acute management of moderate-to-severe traumatic brain injury. J Neurotrauma 2016;33:1461-78.

8. Boat TF, Chao SM, O'Neill PH. From waste to value in health care. JAMA 2008;299:568-71.

9. Reilly BM, Evans AT. Much ado about (doing) nothing. Ann Intern Med 2009:150:270-1.

10. Berwick DM, Hackbarth AD. Eliminating waste in US health care. JAMA 2012;307:1513-6.

11. Choosing Wisely Canada, 2015. Available: https:// choosingwiselycanada.org/ [Accessed 19 Nov 2018].

12. Morgan DJ, Dhruva SS, Wright SM, et al. 2016 update on medical overuse: a systematic review. JAMA Intern Med 2016;176:1687-92.

13. Berwick DM. Avoiding overuse-the next quality frontier. The Lancet 2017:390:102-4.

14. Brownlee S, Chalkidou K, Doust J, et al. Evidence for overuse of medical services around the world. Lancet 2017;390:156-68.

15. Saini V, Brownlee S, Elshaug AG, et al. Addressing overuse and underuse around the world. The Lancet 2017;390:105-7.

16. Fisher ES, Wennberg DE, Stukel TA, et al. The implications of regional variations in Medicare spending. Part 2: health outcomes and satisfaction with care. Ann Intern Med 2003;138:288-98.

17. Niven DJ, Mrklas KJ, Holodinsky JK, et al. Towards understanding the de-adoption of low-value clinical practices: a scoping review. BMC Med 2015;13:255.

18. Emanuel EJ, Fuchs VR. The perfect storm of overutilization. JAMA 2008;299:2789-91.

19. Carney N, Totten AM, O'Reilly C, et al. Guidelines for the management of severe traumatic brain injury, fourth edition. Neurosurgery 2017;80:6-15.

20. Moore L, Lauzier F, Tardif PA, et al. Low-Value clinical practices in injury care: a scoping review and expert consultation survey. $J$ Trauma Acute Care Surg 2019.

21. Lei J, Gao G-Y, Jiang J-Y. Is management of acute traumatic brain injury effective? A literature review of published Cochrane systematic reviews. Chin J Traumatol 2012;15:17-22.
22. Lu J, Gary KW, Copolillo A, et al. Randomized controlled trials in adult traumatic brain injury: a review of compliance to consort statement. Arch Phys Med Rehabil 2015;96:702-14.

23. Synnot $A$, Bragge $P$, Lunny $C$, et al. The currency, completeness and quality of systematic reviews of acute management of moderate to severe traumatic brain injury: a comprehensive evidence MAP. PLOS One 2018;13:e0198676.

24. Higgins J, Green S. Cochrane Handbook for systematic reviews of interventions version 5.1.0. The Cochrane Collaboration, 2011.

25. Smith V, Devane D, Begley CM, et al. Methodology in conducting a systematic review of systematic reviews of healthcare interventions. BMC Med Res Methodol 2011;11:15.

26. The Joanna Briggs Institute. Joanna Briggs Institute Reviewers' Manual: 2014 edition / Supplement. The Joanna Briggs Institute 2014.

27. Moher D, Shamseer L, Clarke M, et al. Preferred reporting items for systematic review and meta-analysis protocols (PRISMA-P) 2015 statement. Syst Rev 2015;4:1.

28. Stone PW. Popping the (PICO) question in research and evidencebased practice. Appl Nurs Res 2002;15:197-8.

29. Marshall IJ, Marshall R, Wallace BC, et al. Rapid reviews may produce different results to systematic reviews: a metaepidemiological study. J Clin Epidemiol 2019;109:30-41.

30. Bramer WM, Rethlefsen ML, Kleijnen J, et al. Optimal database combinations for literature searches in systematic reviews: a prospective exploratory study. Syst Rev 2017;6:245.

31. Epistemonikos. Epistemonikos n.d. Available: http://www. epistemonikos.org/en/ [Accessed 19 Nov 2018]

32. Centre for Reviews and Dissemination. Prospero. Available: https:// www.crd.york.ac.uk/PROSPERO/ [Accessed 19 Nov 2018]

33. Horton L, Rhodes J, Wilson L. Randomized controlled trials in adult traumatic brain injury: a systematic review on the use and reporting of clinical outcome assessments. J Neurotrauma 2018;35:2005-14.

34. Bramer WM, Giustini D, de Jonge GB, et al. De-duplication of database search results for systematic reviews in endnote. J Med Libr Assoc 2016;104:240-3.

35. de Oliveira IR, Santos-Jesus R, Po ALW, et al. Extracting numerical data from published reports of pharmacokinetics investigations: method description and validation. Fundam Clin Pharmacol 2003;17:471-2.

36. Robson RC, Pham Ba', Hwee J, et al. Few studies exist examining methods for selecting studies, abstracting data, and appraising quality in a systematic review. J Clin Epidemiol 2019;106:121-35.

37. Shea BJ, Reeves BC, Wells G, et al. AMSTAR 2: a critical appraisal tool for systematic reviews that include randomised or nonrandomised studies of healthcare interventions, or both. $B M J$ 2017;358.

38. Lunny C, Brennan SE, McDonald S, et al. Toward a comprehensive evidence map of overview of systematic review methods: paper 2-risk of bias assessment; synthesis, presentation and summary of the findings; and assessment of the certainty of the evidence. Syst Rev 2018;7:159. 